\title{
Evaluating the Initiation of Novel Oral Anticoagulants in Medicare Beneficiaries
}

\author{
Seo Hyon Baik, PhD; Inmaculada Hernandez, PharmD; and Yuting Zhang, PhD
}

\begin{abstract}
BACKGROUND: As alternatives to warfarin, 2 novel oral anticoagulants (NOACs), dabigatran and rivaroxaban, were approved in 2010 and 2011 to prevent stroke and other thromboembolic events in patients with atrial fibrillation. It is unclear how patient characteristics are associated with the initiation of anticoagulants.
\end{abstract}

OBJECTIVE: To evaluate how patient demographics, clinical characteristics, types of insurance, and patient out-of-pocket spending affect the initiation of warfarin and 2 NOACs-dabigatran and rivaroxaban.

METHODS: We used pharmacy claims data from a $5 \%$ random sample of Medicare beneficiaries to identify patients who were newly diagnosed with atrial fibrillation between 0ctober 1, 2010, and 0ctober 31, 2012, and who were prescribed an oral anticoagulant within 60 days of diagnosis. We identified key predictors of initiation of NOACs using a multinomial logistic regression model with generalized logit link.

RESULTS: Patients who were black and who had a history of acute myocardial infarction, stroke or transient ischemic attack, chronic kidney disease, or congestive heart failure were significantly associated with lower odds of receiving NOACs compared with warfarin. Age greater than 65 years, a history of hypertension, and use of nonsteroidal anti-inflammatory drugs were positively associated with the initiation of NOACs. Rivaroxaban was most likely to be initiated among women, followed by warfarin and dabigatran. Individuals receiving a low-income subsidy were more likely to initiate warfarin than NOACs, even though they paid little copayment. Individuals with supplemental Part D drug coverage, such as national Programs for All-Inclusive Care for the Elderly or employer-sponsored plans, were more likely to initiate NOACs compared with warfarin.

CONCLUSIONS: We found that race, sex, type of Part D plans, and some clinical conditions were associated with the initiation of NOACs relative to warfarin. But patient demographic and clinical characteristics did not appear to affect which particular NOAC patients initiated.

J Manag Care Spec Pharm. 2016;22(3):281-92

Copyright $\odot 2016$, Academy of Managed Care Pharmacy. All rights reserved.

\section{What is already known about this subject}

Dabigatran and rivaroxaban do not require routine blood monitoring and have fewer interactions than warfarin.

In their respective clinical trials, dabigatran $150 \mathrm{mg}$ and rivaroxaban $20 \mathrm{mg}$ were found to be as effective as warfarin in preventing stroke and systemic embolism and similar in the rates of major bleeding.

However, both dabigatran and rivaroxaban need to be used with caution in patients with renal impairment.

\section{What this study adds}

Patients who were black and who had a history of acute myocardial infarction, stroke or transient ischemic attack, chronic kidney disease, or congestive heart failure were significantly associated with lower odds of receiving novel oral anticoagulants (NOACs) compared with warfarin.

Age greater than 65 years, a history of hypertension, and use of nonsteroidal anti-inflammatory drugs were positively associated with the initiation of NOACs.

Individuals receiving a low-income subsidy were more likely to initiate warfarin than NOACs, even though they paid little copayment. Individuals with supplemental Part D drug coverage, such as national Programs for All-inclusive Care for the Elderly or employer-sponsored plans, were more likely to initiate NOACs compared with warfarin

$\Lambda$ trial fibrillation affects between 2.7 million and 6.1 million American adults and is estimated to result in more than 99,000 deaths per year in the United States. ${ }^{1}$ The U.S. Food and Drug Administration approved the use of dabigatran (brand name Pradaxa) $75 \mathrm{mg}$ and $150 \mathrm{mg}$ twice-a-day regimens and rivaroxaban (brand name Xarelto) $15 \mathrm{mg}$ and $20 \mathrm{mg}$ once-a-day regimens to reduce the risk of stroke and systemic embolism among nonvalvular atrial fibrillation patients in October 2010 and November 2011, respectively. ${ }^{2,3}$ Warfarin (brand name Coumadin) was the only available oral anticoagulant before the approval of these 2 novel oral anticoagulants (NOACs). Dabigatran $150 \mathrm{mg}$ and rivaroxaban $20 \mathrm{mg}$ were found to be as effective as warfarin in preventing stroke and systemic embolism and similar in the rates of major bleeding. ${ }^{4,5}$ Dabigatran $150 \mathrm{mg}$ and rivaroxaban $20 \mathrm{mg}$ were demonstrated to have a lower rate of intracranial bleeding than warfarin but higher risks of gastrointestinal bleeding., ${ }^{5,6}$

NOACs are much more convenient to use than warfarin. Dosing of warfarin needs to be adjusted by the international normalized ratio (INR), ${ }^{7}$ which requires routine blood testing. In addition, warfarin interacts with many medications and foods. In contrast, NOACs are administered once or twice a day at a fixed dosage, do not require routine INR blood monitoring, and have fewer interactions with other medications. ${ }^{4}$ Both dabigatran $150 \mathrm{mg}$ and rivaroxaban $20 \mathrm{mg}$ have been shown to be cost-effective alternatives to warfarin for 
atrial fibrillation patients at the threshold value of $\$ 50,000$ per quality-adjusted life-year. ${ }^{8,9}$ As a result, dabigatran and rivaroxaban are acclaimed to be potential alternatives to warfarin for patients who are unable to comply with warfarin and its frequent dosage adjustments. However, the drug cost and thus patients' out-of-pocket cost for both dabigatran and rivaroxaban are much higher than those for warfarin. For instance, the Medicare gross cost for a month supply of dabigatran and rivaroxaban was $\$ 228$ and $\$ 240$, compared with $\$ 14$ for warfarin. Besides, these 2 NOACs need to be used with caution in patients with renal impairment.

Given the initial promise of NOACs and the subsequent concerns about their limitations, it is relevant to study which factors affect the initiation of anticoagulants in the real-world setting. Since the prevalence of atrial fibrillation increases with age, the use of anticoagulants is especially high among Medicare beneficiaries. The recent availability of 2012 Medicare Part D data makes it possible to assess the association between patient characteristics and the initiation of 3 anticoagulants among this population. In this study, we evaluated how patient demographics, clinical characteristics, type of insurance, and out-of-pocket expenses were associated with the initiation of anticoagulant treatment among patients newly diagnosed with atrial fibrillation.

\section{Methods}

\section{Data Source and Study Cohort}

To evaluate how NOACs were adopted in the real-world setting, we used a 5\% random sample of Medicare beneficiaries between 2010 and 2012 (the most recent data available) from the Centers for Medicare \& Medicaid Services (CMS). The study cohort included beneficiaries who were newly diagnosed with atrial fibrillation between October 1, 2010, and October 31, 2012, and initiated warfarin, dabigatran, or rivaroxaban within 60 days of the diagnosis. We excluded those who filled a prescription for more than 1 anticoagulant in this time window (Figure 1). We used the CMS Chronic Condition Warehouse (CCW) indicator that traced the first diagnosis date back to January 1, 1999. A diagnosis of atrial fibrillation was defined as having 1 inpatient or 2 outpatient claims with primary or secondary International Classification of Diseases, Ninth Revision, Clinical Modification (ICD-9-CM) diagnosis code 427.31. ${ }^{10}$

\section{Outcome and Covariates}

To assess factors associated with the initiation of anticoagulants among those newly diagnosed with atrial fibrillation, we defined a categorical variable, in which each category represents 1 of the 3 anticoagulants a patient initiated within 60 days of the atrial fibrillation diagnosis.

We defined sets of covariates and evaluated whether they affected the initiation of newer anticoagulants among those newly diagnosed with atrial fibrillation: time at the initiation, patient demographics, clinical characteristics, and insurance status and out-of-pocket expenses.

\section{FIGURE 1 Deposition of Study Population}

Medicare beneficiaries diagnosed with atrial fibrillation between October 1, 2010, and October 31, 2012 $\mathrm{N}=115,809$

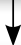

Medicare atrial fibrillation beneficiaries with at least 1 claim of anticoagulants-warfarin, dabigatran, rivaroxaban $\mathrm{N}=33,024$

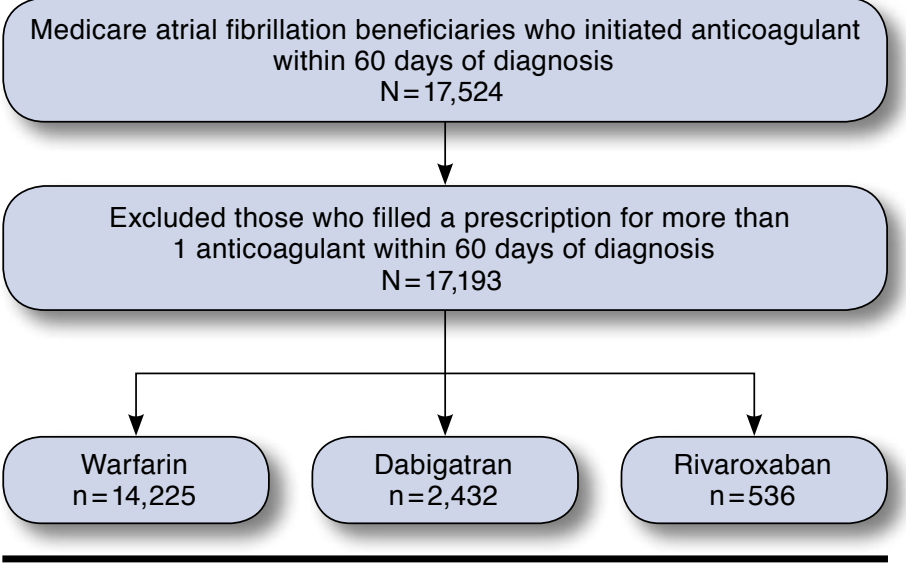

Time at the Initiation. It is very likely that initiation patterns of NOACs may change with time. Although warfarin has been on the market since 1964, dabigatran and rivaroxaban were approved on October 19, 2010, and November 4, 2011, respectively. That is, only warfarin and dabigatran were marketed at the beginning of the study and rivaroxaban became available on November 4, 2011. We defined 6 time indicators depending on the time of initiating an anticoagulant: October 1, 2010-March 3, 2011; March 4, 2011-July 3, 2011; July 4, 2011-November 3, 2011; November 4, 2011-March 3, 2012; March 4, 2012-July 3, 2012; and July 4, 2012-December 31,2012 . Including these time periods in the model enabled us to estimate how time affects the use of anticoagulants.

Patient Demographics. Demographic characteristics included age, sex, race (White, Black, Hispanic, Asian, Other) and region of residence. We categorized the study sample into nonelderly (age 64 years or younger) and elderly (age 65 years or older) groups according to their age at the end of the year when they initiated the drug of interest. The nonelderly group included those who had Social Security disability benefits for at least 24 months or permanent kidney failure requiring dialysis or a kidney transplant. Moreover, we split the elderly group into age 65 years- 74 years and 75 years or older because of potential treatment-by-age interactions. We divided the ZIP code of 
residence into 4 geographic areas: South (Alabama, Arkansas, Delaware, District of Columbia, Florida, Georgia, Kentucky, Louisiana, Maryland, Mississippi, North Carolina, Oklahoma, South Carolina, Tennessee, Texas, Virginia, and West Virginia); West (Alaska, Arizona, California, Colorado, Hawaii, Idaho, Montana, Nevada, New Mexico, Oregon, Utah, Washington, and Wyoming); Midwest (Illinois, Indiana, Iowa, Kansas, Michigan, Minnesota, Missouri, Nebraska, North Dakota, Ohio, South Dakota, and Wisconsin); and Northeast (Connecticut, Maine, Massachusetts, New Hampshire, New Jersey, New York, Pennsylvania, Rhode Island, and Vermont).

Clinical Characteristics. Patient clinical characteristics include history of acute myocardial infarction (AMI) or ischemic heart disease, stroke or transient ischemic attack, diabetes, chronic kidney disease, congestive heart failure (CHF), hypertension, hypothyroidism, cancers (breast, colorectal, prostate, lung, endometrial), number of other CMS predefined CCW priority chronic conditions, death during the study period, use of other medications such as nonsteroidal anti-inflammatory drugs (NSAIDs) or antiplatelet agents, and prescription drug hierarchical condition category (CMS-RxHCC) score. We used the CCW indicator that traces the first diagnosis of these conditions date back to January 1, 1999 (ICD-9-CM codes are listed in the Appendix, available in the online article). The number of other CMS predefined CCW priority chronic conditions includes Alzheimer's disease-related disorders or senile dementia, anemia, asthma, benign prostatic hyperplasia, cataract, chronic obstructive pulmonary disease, depression, glaucoma, hip or pelvic fracture, hyperlipidemia, osteoporosis, and rheumatoid arthritis or osteoarthritis. Atrial fibrillation and previously defined conditions were excluded in order to avoid including each condition in more than 1 covariate. ${ }^{11}$

Use of NSAIDs was defined as having at least 1 prescription for diclofenac, ibuprofen, naproxen, ketoprofen, fenoprofen, flurbiprofen, piroxicam, meloxicam, mefenamic acid, or indomethacin 60 days before initiating anticoagulants. The use of antiplatelet agents was defined as having at least 1 pharmacy claim for aspirin, clopidogrel, prasugrel, dipyridamol, ticlopidine, and ticagrelor 60 days before initiating anticoagulants. CMS-RxHCC is a prospective risk score calculated by using a patient's prioryear diagnoses; a higher value indicates worse health status. ${ }^{12}$

Insurance Status and Out-of-Pocket Expenses. To study the effect of insurance types and patients' out-of-pocket expenses at the time of initiating an anticoagulant, we first divided our study sample into 2 groups according to patients' prescription drug insurance status: those who enrolled in stand-alone Part D plans and supplemental Part D drug plans, including national Programs of All-Inclusive Care for the Elderly (PACE) or employer-sponsored plan enrollees. Generally, patients who enrolled in national PACE or employer-sponsored plans have additional and more generous drug coverage than the stand- alone Part D plans because they fulfill their medical needs through managed and comprehensive care. ${ }^{13}$

We further broke down stand-alone Part D plan enrollees based on patients' cost-sharing status: those who are eligible for both Medicare and Medicaid coverage (dual-eligibles), those who are not dually eligible but receive a low-income subsidy for drugs (non-dual LIS), and non-LIS. Those who belong to either dualeligible or non-dual LIS receive a subsidy from the government and are therefore responsible for zero to $15 \%$ of the total drug price; on average, they paid at most $\$ 2$ for warfarin, $\$ 13$ for dabigatran, and $\$ 17$ for rivaroxaban. Patients' out-of-pocket expenses for non-LIS beneficiaries are much higher and also subject to the standard Part D coverage benefit phase.

We further divided non-LIS beneficiaries into several groups according to the standard Part D coverage benefit phase at the time of drug initiation: initial coverage phase, coverage gap phase, and catastrophic phase. The standard Part D coverage includes a small deductible, about $25 \%$ coinsurance in the initial coverage period, $100 \%$ copayment in the coverage gap, and $5 \%$ in the catastrophic period. Furthermore, starting in 2011, patients received a $50 \%$ manufacturer discount for brand-name drugs in the coverage gap; thus, the patients' copayment for NOACs in the coverage gap was about $50 \%$ of the total drug price for the majority of our study period. In summary, patients' out-of-pocket cost toward the initiation of anticoagulants varies by drug coverage benefit phases as well as patients' cost-sharing status.

\section{Statistical Model}

To evaluate the initiation of anticoagulants, we conducted a multinomial logistic regression with generalized logit link function to model the probability of initiating NOACs against warfarin. The multinomial logistic regression approach enabled us to compare more than 2 study groups through a combination of binary logistic regressions. An advantage of using a multinomial logistic regression over a series of binary logistic regressions is that we are able to draw conclusions across 3 anticoagulants through direct comparisons. We included all covariates and two-way interactions between the time variable and each covariate in the multinomial logistic regression.

Moreover, we conducted a stepwise variable selection method to identify a set of covariates that were significantly associated with the initiation of anticoagulants. Thirteen out of 37 variables were selected. We reported the estimated coefficients of selected covariates for each of the 2 comparisons and plotted estimated odds ratios of initiating 1 anticoagulant against the others, adjusted for the covariates listed above. We did not include the risk score CHADS2 in our model because it is a linear combination of age and 4 chronic conditions: history of CHF, hypertension, diabetes, and stroke that were already included in the model as separate covariates. We included them separately because different conditions could be differently associated with the initiation of anticoagulants. All analyses were conducted 
using commercially available statistical software (SAS software package 9.4 [SAS Institute, Cary, NC]).

\section{Results}

\section{Baseline Characteristics}

Table 1 presents differences in patient characteristics across 3 anticoagulant groups. The age distribution of patients who initiated NOACs differs from that of warfarin users. About 95\% of NOAC users were aged 65 years or older, whereas those aged 65 years or older accounted for about $89 \%$ of warfarin users. The proportion of minorities among NOAC users was smaller than that among warfarin users (10\%-13\% vs. $18 \%$ ).

Among our study population of atrial fibrillation patients, the most frequent chronic condition was hypertension, with a prevalence of more than $87 \%$ of the study population, followed by ischemic heart disease, CHF, diabetes, chronic kidney disease, hypothyroidism, stroke or transient ischemic attack, cancers, and AMI. In terms of the patients' health condition, NOAC users were relatively healthier than warfarin users. They had fewer chronic conditions and a lower CHADS2 score, drug risk score, and rate of death.

The proportion of stand-alone Part D enrollees was relatively lower among NOAC users (86\%-88\% vs. 93\%). Moreover, the proportion of dual-eligibles or non-dual LIS recipients among stand-alone Part D enrollees was smaller among NOAC users (22\%-27\% vs. $40 \%)$ than warfarin users. Whereas the patients' out-of-pocket expenses toward anticoagulants was relatively lower among dual-eligibles or non-dual LIS recipients (who paid at most $\$ 13$ for dabigatran, $\$ 17$ for rivaroxaban, and $\$ 2$ for warfarin), the patients' out-of-pocket cost among nonsubsidy recipients was relatively higher and also subjected to the standard Part D drug coverage benefit phase at the time of filling a prescription. The proportion of patients who used NSAIDs before initiating anticoagulants was higher among NOAC users, but the proportion of antiplatelet users was similar.

\section{The Effect of Time at the Initiation}

After controlling for patient characteristics and insurance status, the odds of initiating dabigatran compared with warfarin were $50 \%$ lower ( $95 \%$ confidence interval $[\mathrm{CI}]=39 \%-58 \%$ ) in the first 4 months (October 2010-February 2011) after dabigatran introduction than in the second half of 2012. However, atrial fibrillation patients were more likely to initiate dabigatran compared with warfarin between March 2011 and June 2012 than in the second half of 2012. The odds of initiating rivaroxaban, as compared with initiating dabigatran or warfarin, increased since the market introduction of rivaroxaban until the end of our study period (December 31, 2012). For example, the odds of initiating rivaroxaban compared with dabigatran in the first 4 months after rivaroxaban's introduction were $80 \%$ lower (95\% CI $=74 \%-85 \%$ ) than in the second half of 2012 . These results were expected and represent the normal diffusion of a drug in a market after approval.

\section{The Effect of Patient Demographics}

Table 2 includes the estimated coefficients of patient demographics, and Figures 2, 3, and 4 compare the odds of initiating 1 anticoagulant against the other at each level of selected covariates. We observed that the use of anticoagulants was associated with patients' sex, age, and race. Women were more likely to initiate rivaroxaban, followed by warfarin and dabigatran. Patients older than 65 years were more likely to initiate 2 NOACs compared with warfarin, but age didn't affect whether rivaroxaban or dabigatran was initiated. Patients who were black were the least likely to initiate NOACs compared with other racial groups.

\section{The Effect of Type of Insurance and Patients' Out-of-Pocket Cost}

Individuals enrolled in national PACE or employer-sponsored plans were more likely to initiate new anticoagulants relative to warfarin, compared with individuals enrolled in stand-alone Part D plans (Table 2 and Figures 2, 3, and 4). Unlike standalone Part D, national PACE or employer-sponsored plans often cover both prescription drugs and medical care services and are more likely to use managed care management. We also found that individuals receiving an LIS, for example, with Medicaid coverage or a federal LIS for prescription drugs were less likely to initiate new anticoagulants. Moreover, we compared the initiation of anticoagulants among those enrolled in stand-alone Part D plans without receiving an LIS. Their out-of-pocket expenses varied depending on which benefit phase they were in at the time of initiating an anticoagulant. However, the outof-pocket expenses at the time of initiation did not appear to affect the type of anticoagulant the patient initiated.

\section{The Effect of Health Conditions}

We found that patients' preexisting chronic conditions affected the initiation of an anticoagulant. A history of AMI, stroke or transient ischemic attack, chronic kidney disease, CHF, or hypertension were key chronic conditions to predict which anticoagulant to initiate, and different conditions were differently associated with the use of anticoagulants. Warfarin was most likely to be prescribed among those with a history of AMI, stroke or transient ischemic attack, chronic kidney disease, or CHF; whereas those with a history of hypertension were more likely to initiate NOACs but did not differentiate one from the other. We also observed that previous use of NSAIDs affected the initiation of anticoagulants. Patients who filled a prescription for NSAIDs in the 60 days before anticoagulant initiation were significantly more likely to initiate NOACs than warfarin. However, use of antiplatelet agents did not affect the initiation of NOACs (Table 2 and Figures 1, 2, and 3).

\section{Discussion}

In this study, we examined how initiation of anticoagulants was associated with patient demographics, clinical conditions, 


\begin{tabular}{|c|c|c|c|c|c|}
\hline & & Dabigatran & Rivaroxaban & Warfarin & \\
\hline & & $\mathrm{n}=2,432$ & $\mathrm{n}=536$ & $\mathrm{n}=14,225$ & $P$ Value ${ }^{a}$ \\
\hline \multirow[t]{6}{*}{ Time at initiation (\%) } & October 1, 2010-March 3, 2011 & 8.18 & 0.00 & 19.17 & \multirow[t]{6}{*}{$<0.001$} \\
\hline & March 4, 2011-July 3, 2011 & 16.90 & 0.00 & 16.40 & \\
\hline & July 4, 2011-November 3, 2011 & 20.23 & 0.19 & 14.65 & \\
\hline & November 4, 2011-March 3, 2012 & 22.45 & 11.75 & 17.91 & \\
\hline & March 4, 2012-July 3, 2012 & 18.30 & 34.14 & 15.71 & \\
\hline & July 4, 2012-December 31, 2012 & 13.94 & 53.92 & 16.16 & \\
\hline \multirow[t]{4}{*}{ Region (\%) } & Midwest & 20.71 & 21.46 & 26.00 & \multirow[t]{4}{*}{$<0.001$} \\
\hline & Northeast & 21.82 & 15.30 & 22.31 & \\
\hline & South & 41.99 & 51.87 & 36.38 & \\
\hline & West & 15.48 & 11.38 & 15.31 & \\
\hline \multirow[t]{3}{*}{ Age, years (\%) } & 65 or younger & 5.26 & 5.22 & 11.04 & \multirow{3}{*}{$<0.001$} \\
\hline & $65-74$ & 40.83 & 36.19 & 33.88 & \\
\hline & 75 or older & 53.91 & 58.58 & 55.08 & \\
\hline Sex (\%) & Women & 55.51 & 61.75 & 58.19 & 0.009 \\
\hline \multirow[t]{5}{*}{ Race (\%) } & White & 87.25 & 89.55 & 81.90 & \multirow{5}{*}{$<0.001$} \\
\hline & Black & 4.89 & 4.66 & 9.14 & \\
\hline & Hispanic & 4.11 & 3.17 & 5.64 & \\
\hline & Asian & 1.97 & 1.68 & 1.93 & \\
\hline & Other & 1.77 & 0.93 & 1.39 & \\
\hline Death (\%) & & 3.70 & 2.80 & 8.27 & $<0.001$ \\
\hline \multirow{6}{*}{$\begin{array}{l}\text { Patient's insurance, cost-sharing status, } \\
\text { and drug coverage benefit at the } \\
\text { initiation of an anticoagulant (\%) }\end{array}$} & Dual-eligibles & 21.92 & 18.10 & 33.08 & \multirow{6}{*}{$<0.001$} \\
\hline & Non-dual LIS & 4.85 & 4.48 & 6.55 & \\
\hline & Non-LIS in the initial phase & 55.39 & 58.96 & 47.45 & \\
\hline & Non-LIS in the coverage gap phase & 4.98 & 4.85 & 4.78 & \\
\hline & Non-LIS in the catastrophic phase & 1.03 & 0.56 & 0.78 & \\
\hline & National PACE or employer-sponsored & 11.84 & 13.06 & 7.35 & \\
\hline \multirow[t]{9}{*}{ Preexisting chronic conditions (\%) } & Acute myocardial infarction & 5.88 & 7.09 & 8.65 & $<0.001$ \\
\hline & Ischemic heart disease & 61.92 & 64.93 & 64.93 & 0.016 \\
\hline & Stroke/transient ischemic attack & 19.04 & 15.11 & 23.54 & $<0.001$ \\
\hline & Diabetes & 38.73 & 40.67 & 45.64 & $<0.001$ \\
\hline & Chronic kidney disease & 23.81 & 24.81 & 35.27 & $<0.001$ \\
\hline & Congestive heart failure & 40.75 & 36.57 & 52.79 & $<0.001$ \\
\hline & Hypertension & 88.86 & 90.86 & 87.58 & 0.020 \\
\hline & Hypothyroidism & 21.42 & 23.88 & 21.74 & 0.454 \\
\hline & Cancers $^{b}$ & 14.06 & 15.86 & 14.81 & 0.477 \\
\hline
\end{tabular}

type of insurance, and out-of-pocket expenses. Among demographic characteristics, women were associated with higher odds of initiating rivaroxaban but lower odds of receiving dabigatran compared with warfarin. Patients older than 65 years had significantly higher odds of receiving NOACs but did not differentiate one from the other. Black patients were least likely to be prescribed NOACs.

Some patients' health conditions were inseparable from the initiation of NOACs. Warfarin was more likely to be prescribed over NOACs among patients who had been diagnosed with AMI, stroke or transient ischemic attack, chronic kidney disease, or $\mathrm{CHF}$ at the time of initiating an anticoagulant. In contrast, patients who had a history of hypertension or who used NSAIDs were more likely to be prescribed NOACs.
Patients who enrolled in plans offering supplemental Part D drug coverage such as national PACE or employer-sponsored plans were more likely to be prescribed NOACs than those enrolled in stand-alone Part D plans. Among stand-alone Part D plan enrollees, lower out-of-pocket expenses toward NOACs did not increase the odds of initiating NOACs. Even a little copayment for NOACs—at most, $\$ 13$ for dabigatran and $\$ 17$ for rivaroxaban-may still be cost-prohibitive, especially compared with $\$ 2$ for warfarin among patients receiving an LIS. These patients do not completely remove costs as a consideration in making a decision. Moreover, prescribers may not be aware that the copayment for patients receiving an LIS are relatively low for NOACs, and they may have the perception that NOACs are still not affordable for most patients. 
TABLE 1 Comparisons of Baseline Characteristics Among Beneficiaries Who Were Newly Diagnosed with Atrial Fibrillation and Initiated Dabigatran, Rivaroxaban, or Warfarin Within 60 Days of Diagnosis, by Treatment (continued)

\begin{tabular}{|c|c|c|c|c|c|}
\hline & & Dabigatran & Rivaroxaban & Warfarin & \multirow[b]{2}{*}{$P$ Value } \\
\hline & & $\mathrm{n}=2,432$ & $\mathrm{n}=536$ & $\mathrm{n}=14,225$ & \\
\hline \multirow{3}{*}{$\begin{array}{l}\text { Number of } 12 \text { CCW chronic } \\
\text { conditions }{ }^{c}(\%)\end{array}$} & 3 or fewer & 42.68 & 38.99 & 38.56 & \multirow[t]{3}{*}{$<0.001$} \\
\hline & $4-6$ & 45.23 & 46.64 & 44.75 & \\
\hline & 7 or more & 12.09 & 14.37 & 16.70 & \\
\hline \multirow[t]{3}{*}{ CHADS2 score (\%) } & 1 or fewer & 23.56 & 21.27 & 18.52 & \multirow[t]{3}{*}{$<0.001$} \\
\hline & $2-3$ & 52.30 & 57.28 & 49.85 & \\
\hline & 4 or more & 24.14 & 21.46 & 31.63 & \\
\hline \multirow[t]{2}{*}{ Previous use of other medications ${ }^{\mathrm{d}}(\%)$} & Antiplatelet ${ }^{\mathrm{e}}$ & 7.07 & 8.58 & 4.70 & \multirow[t]{2}{*}{$<0.001$} \\
\hline & NSAID $^{f}$ & 7.28 & 6.90 & 7.35 & \\
\hline Gross drug cost (mean $[ \pm \mathrm{SD}])$ & & $264.34[125.38]$ & $269.37[128.54]$ & $12.37 \quad[14.7]$ & $<0.001$ \\
\hline \multirow{6}{*}{$\begin{array}{l}\text { Patient pay amount toward an } \\
\text { anticoagulant (mean }[ \pm S D] \text { ) }\end{array}$} & Dual-eligibles & $3.33 \quad[2.15]$ & {$[2.52]$} & $0.96 \quad[1.01]$ & \\
\hline & Non-dual LIS & $12.51 \quad[17.29]$ & $17.87 \quad[27.3]$ & $1.96 \quad[1.53]$ & \multirow[t]{5}{*}{$<0.001$} \\
\hline & Non-LIS in the initial phase & $82.25 \quad[59.52]$ & $77.15 \quad[55.24]$ & $7.57 \quad[10.44]$ & \\
\hline & Non-LIS in the coverage gap phase & $143.61 \quad[80.02]$ & $109.1 \quad[27.15]$ & 9.89 [12.08] & \\
\hline & Non-LIS in the catastrophic phase & $11.79 \quad[4.29]$ & $12.09 \quad[0.00]$ & $2.76 \quad[1.29]$ & \\
\hline & National PACE or employer-sponsored & $56.62 \quad[52.41]$ & $65.81[58.77]$ & $8.07 \quad[10.35]$ & \\
\hline CMS-RxHCC risk scores (mean $[ \pm S D]$ ) & & $0.94 \quad[0.36]$ & $0.96 \quad[0.33]$ & $1.00 \quad[0.43]$ & $<0.001$ \\
\hline
\end{tabular}

Notes: CHADS2 score is a prediction measure of the risk of stroke in patients with atrial fibrillation in which congestive heart failure, hypertension, age $\geq 75$ years, and diabetes are assigned 1 point. Previous stroke or transient ischemic attack is assigned 2 points. The score is calculated as the sum of all points for a given patient. The number of other comorbidities has been calculated as the sum of a previous history of Alzheimer's disease, related disorders or senile dementia, anemia, asthma, benign prostatic hyperplasia, cataract, chronic obstructive pulmonary disease, depression, hip or pelvic fracture, glaucoma, hyperlipidemia, osteoporosis, and rheumatoid arthritis or osteoarthritis.

${ }^{a}$ We tested differences using chi-square tests for categorical variables and F-tests for numerical variables.

${ }^{b}$ Cancers include breast, colorectal, prostate, lung, and endometrial.

¿12 CCW chronic conditions include Alzheimer's disease, related disorders or senile dementia, anemia, asthma, benign prostatic hyperplasia, cataract, chronic obstructive pulmonary disease, depression, glaucoma, hip or pelvic fracture, hyperlipidemia, osteoporosis, and rheumatoid arthritis or osteoarthritis.

"Previous use of other medications" is defined as any use of either antiplatelet or NSAID within 60 days before the first use of an anticoagulant.

eAntiplatelet includes any drug formulation that includes aspirin, clopidrogrel, prasugrel, ticagrelor, ticlopidine, or dipyridamole.

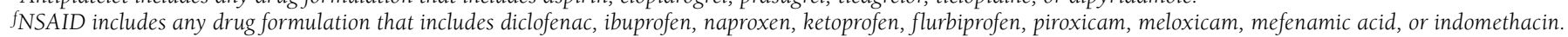
$C C W=$ Chronic Condition Warehouse; LIS=low-income subsidy; NSAID = nonsteroidal anti-inflammatory drug; PACE = Programs of All-Inclusive Care for the Elderly; $R \times H C C=$ prescription drug hierarchical condition category; $S D=$ standard deviation.

Previous studies used either prescription claims data from a large private insurer or atrial fibrillation registry data and investigated how patient characteristics were associated with the likelihood of initiating any of 3 NOACs (dabigatran, rivaroxaban, or apixaban) against warfarin. ${ }^{14-17}$ They found that elderly patients, women, those with lower household income, health maintenance organization enrollees, and those with a higher CHADS2 score, a higher Elixhauser Comorbidity Index, and a higher Anticoagulation and Risk Factors in Atrial Fibrillation score were significantly associated with lower odds of initiating any NOAC..$^{14-17}$ However, they did not compare the initiation of each NOAC against warfarin separately and did not conduct head-to-head comparisons among NOACs ${ }^{15}$ or they included only 1 NOAC. . $^{14,16,17}$

Our study explored the initiating patterns of 3 anticoagulants among Medicare beneficiaries using a multinomial logistic regression model with generalized logit link function that enables all possible pairwise head-to-head comparisons.
Thus, we were able to conclude that rivaroxaban was the most favored anticoagulant among women, followed by warfarin and dabigatran. In addition, because the risk of atrial fibrillation increases with age and the prevalence of atrial fibrillation and use of anticoagulants are higher among Medicare beneficiaries, a Medicare population is more relevant for the study of this research question. Compared with enrollees in previous studies where, at most, $70 \%$ of enrollees were aged 65 years or older, ${ }^{14-16}$ our study population is older; aged 65 years or older accounts for about $90 \%$ of our study population. Moreover, unlike a previous study, ${ }^{15}$ we included the components of the CHADS2 score as separate covariates in our model. The CHADS2 score is a mix of 4 chronic conditions (CHF, hypertension, diabetes, and stroke) plus age 75 years or older. By including them as separate covariates, we found that these conditions affected the initiation of anticoagulants differently. For example, CHF and stroke were negatively associated, but hypertension and age 75 years or older were positively associated with the initiation of NOACs. 
TABLE 2 Estimated Coefficients of Key Covariates from a Multinomial Logistic Regression

\begin{tabular}{l} 
Variable \\
\hline Intercept \\
Time vs. July 4, 2012- \\
December 31, 2012
\end{tabular}

Region vs. Northeast

Age vs. $<65$ years

Sex

Death

Patient's insurance, cost-sharing status, and drug coverage benefit at the initiation of an anticoagulant vs. non-LIS in the initial phase

Preexisting chronic conditions

Previous use of other medications
December 31, 2012

Race vs. White
October 1, 2010-March 3, 2011

October 1, 2010-March 3, 2011

March 4, 2011-July 3, 2011

March 4, 2011-July 3, 2011

July 4, 2011-November 3, 2011

July 4, 2011-November 3, 2011

November 4, 2011-March 3, 2012

November 4, 2011-March 3, 2012

March 4, 2012-July 3, 2012

March 4, 2012-July 3, 2012

Midwest

Midwest

South

South

West

West

65-74

$65-74$

75 or older

75 or older

Women

Women

Black

Black

Hispanic

Hispanic

Asian

Asian

Other

Other

Died

Died

Dual-eligibles

Dual-eligibles

Non-dual LIS

Non-dual LIS

Non-LIS in the coverage gap phase

Non-LIS in the coverage gap phase

Non-LIS in the catastrophic phase

Non-LIS in the catastrophic phase

National PACE or employer-sponsored

National PACE or employer-sponsored

Acute myocardial infarction

Acute myocardial infarction

Stroke/transient ischemic attack

Stroke/transient ischemic attack

Chronic kidney disease

Chronic kidney disease

Congestive heart failure

Congestive heart failure

Hypertension

Hypertension

NSAID

NSAID

Antiplatelet

Antiplatelet

\section{Dab}

NOAC

\begin{tabular}{|l|r|}
\hline Dabigatran & -2.35 \\
\hline Rivaroxaban & -2.95 \\
\hline Dabigatran & -0.69
\end{tabular}

Rivaroxaban $\quad-16.21$

\begin{tabular}{|r|r}
\hline Dabigatran & 0.19 \\
\hline
\end{tabular}

Rivaroxaban

Dabigatran

Rivaroxaban

Dabigatran

Rivaroxaban

Dabigatran

Rivaroxaban

Dabigatran

Rivaroxaban

Dabigatran

Rivaroxaban

Dabigatran

Rivaroxaban

Dabigatran

Rivaroxaban

Dabigatran

Rivaroxaban

Dabigatran

Rivaroxaban

Dabigatran

Rivaroxaban

Dabigatran

Rivaroxaban

Dabigatran

Rivaroxaban

Dabigatran

Rivaroxaban

Dabigatran

Rivaroxaban

Dabigatran

Rivaroxaban

Dabigatran

Rivaroxaban

Dabigatran

Rivaroxaban

Dabigatran

Rivaroxaban

Dabigatran

Rivaroxaban

Dabigatran

Rivaroxaban

Dabigatran

Rivaroxaban

Dabigatran

Rivaroxaban

Dabigatran

Rivaroxaban

Dabigatran

Rivaroxaban

Dabigatran

Rivaroxaban

Dabigatran

Rivaroxaban
0.19
-16.14

0.48

$-5.61$

0.41

$-1.62$

0.31

$-0.44$

-0.28
0.11

0.19

0.83

0.83
-0.02

0.07

0.62

0.53

0.53
0.54

0.54
-0.08

0.24

$-0.38$

$-0.54$

$-0.18$

$-0.15$

0.10

0.10

0.22

$-0.21$

$-0.21$

$-0.68$

$-0.35$

$-0.62$

-0.26
-0.39

$-0.03$

$-0.22$

-0.22
0.35

0.35
-0.49

0.31

$-0.27$

0.07
-0.19

-0.19
-0.53

-0.53
-0.40

$-0.34$

$-0.34$

-0.29
-0.49

0.40

0.58

0.48

0.72

0.16

0.11

\begin{tabular}{|c|c|}
\hline$P$ Value \\
\hline$<0.0001$ \\
$<0.0001$ \\
$<0.0001$
\end{tabular}

EXP (Effect)

0.10

0.0

\begin{tabular}{l}
0.02 \\
0.93 \\
\hline 0.0001
\end{tabular}

$<0.0001$

$<0.0001$

$<0.0001$

$<0.0001$

$<0.0001$

$<0.0001$

0.490

$<0.0001$

0.830

0.700

$<0.0001$

0.100

$<0.0001$

0.010

0.090

0.010

0.000

0.010

0.110

0.570

0.550

0.790

0.210

0.650

$<0.0001$

0.010

$<0.0001$

$<0.0001$

0.010

0.080

0.790

0.320

0.130

0.420

$<0.0001$

0.090

0.010

0.710

0.000

$<0.0001$

$<0.0001$

0.000

$<0.0001$

$<0.0001$

$<0.0001$

$<0.0001$

$<0.0001$

$<0.0001$

0.070

0.560

Note: Variables were selected by the stepwise variable selection method. Thirteen variables were selected among 37 variables.

LIS =low-income subsidy; NOAC=novel oral anticoagulant; NSAID =nonsteroidal anti-inflammatory drug; PACE=Programs of All-Inclusive Care for the Elderly. 
October 1, 2010-March 3, 2011

March 4, 2011-July 3, 2011

July 4, 2011-November 3, 2011

November 4, 2011-March 3, 2012

March 4, 2012-July 3, 2012

Midwest

South

West

Age 65-74

Age $>74$

Female

Black

Hispanic

Asian

Other

Death

Dual-eligibles

Non-dual LIS

Non-LIS in the coverage gap phase

Non-LIS in the catastrophic phase National PACE or employer-sponsored

AMI

Stroke

Kidney disease

CHF

Hypertension

NSAID

Antiplatelet

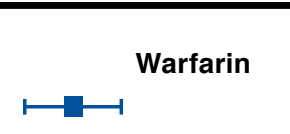

Dabigatran
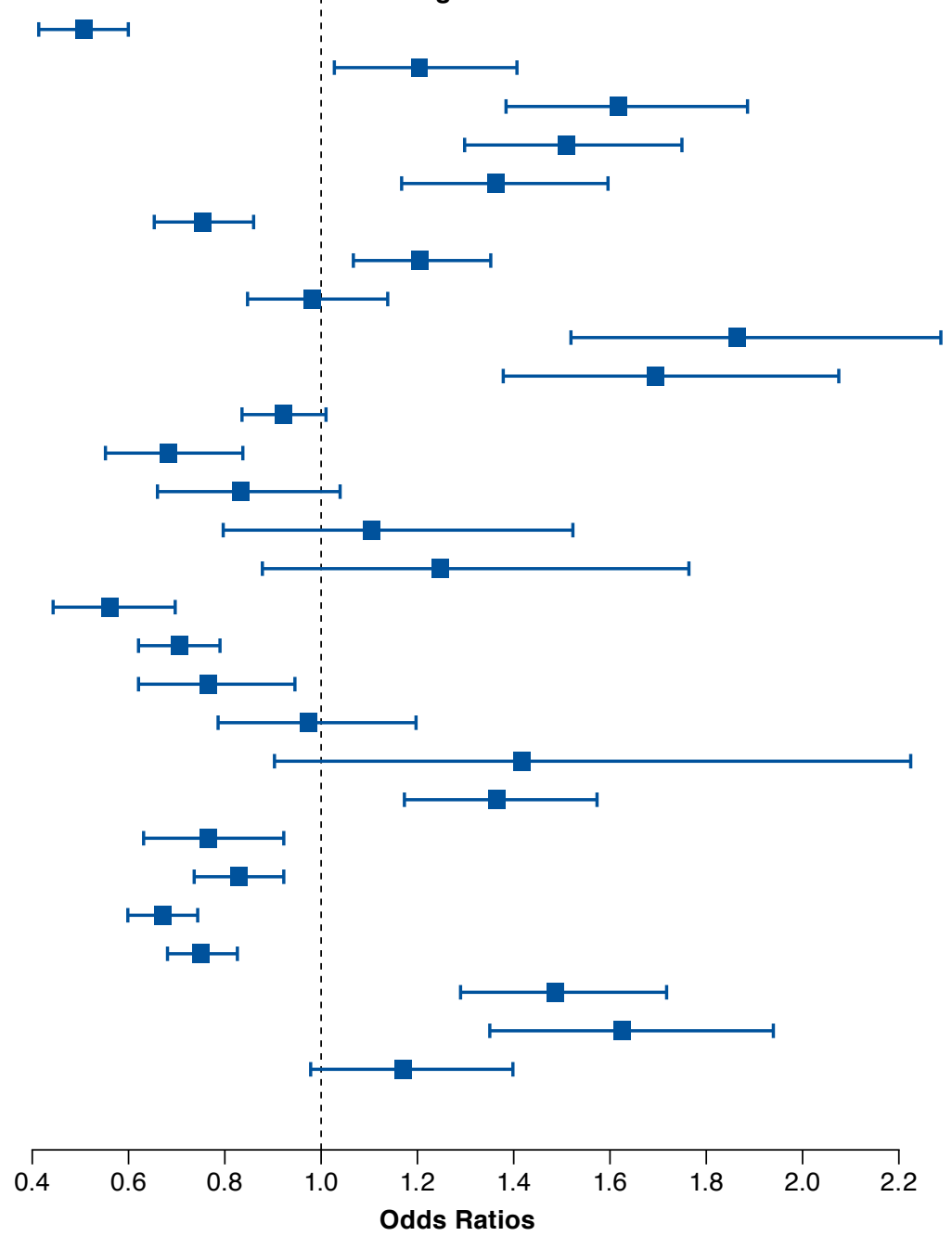

AMI = acute myocardial infarction; $C H F=$ congestive heart failure; LIS = low-income subsidy; NSAID = nonsteroidal anti-inflammatory drug.

Nevertheless, our study has important clinical and policy implications. First, we found that several factors were associated with increased likelihood of initiating NOACs compared with warfarin; however, it was difficult to identify factors differentiating the initiation of rivaroxaban and dabigatran. These results suggest that rivaroxaban and dabigatran are considered interchangeable in the clinical setting. More studies using observational data are needed to compare the effectiveness and safety profile of dabigatran and rivaroxaban in clinical practice and to examine whether there is any subgroup of patients for whom 1 NOAC may be the most favorable choice.

Second, we found that patients using NSAIDs were more likely to initiate dabigatran and rivaroxaban than warfarin.
However, it has been shown that the risk of gastrointestinal bleeding is higher with new anticoagulants than with warfarin. ${ }^{4,5}$ As a result, it is important to warn patients, especially those who are on NSAIDs, about the risk of gastrointestinal hemorrhage with these agents.

\section{Limitations}

Our study has 5 main limitations. First, our study period ends on December 31, 2012. Thus, our findings may not represent current NOAC usage patterns, even among patients on dabigatran and rivaroxaban, because the treatment pattern of NOAC usage is evolving quickly. We also had only 536 patients who initiated rivaroxaban throughout the study period. This number of 
October 1, 2010-March 3, 2011

March 4, 2011-July 3, 2011

July 4, 2011-November 3, 2011

November 4, 2011-March 3, 2012

March 4, 2012-July 3, 2012

Midwest

South

West

Age 65-74

Age $>74$

Female

Black

Hispanic

Asian

Other

Death

Dual-eligibles

Non-dual LIS

Non-LIS in the coverage gap phase

Non-LIS in the catastrophic phase National PACE or employer-sponsored

AMI

Stroke

Kidney disease

CHF

Hypertension

NSAID

Antiplatelet

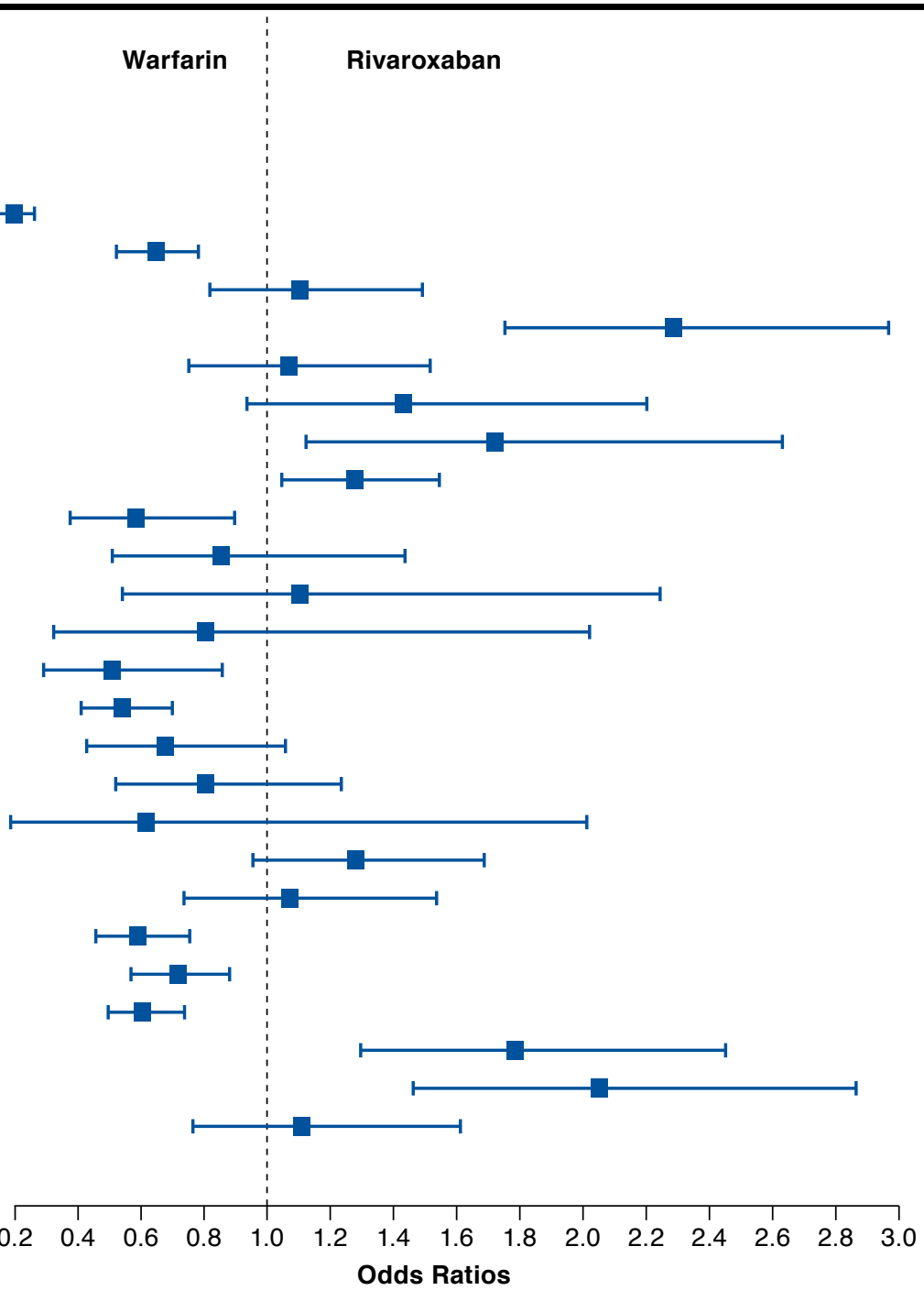

AMI = acute myocardial infarction; $C H F=$ congestive heart failure; $L I S=$ low-income subsidy; NSAID = nonsteroidal anti-inflammatory drug.

rivaroxaban users may not be sufficiently large to draw conclusions about there being no differences between rivaroxaban and each of the other 2 anticoagulants in terms of initiation. This is evidenced in the wide CIs. Moreover, because the study period ended on December 31, 2012, we did not observe the use of apixaban, which was approved to treat atrial fibrillation on December 28, 2012, the third new anticoagulant as a substitute for warfarin. As a result, our study does not evaluate the initiation of anticoagulants when patients have 1 more option of a new anticoagulant. As more recent Part $\mathrm{D}$ data become available, we can expand our analyses to include the other new anticoagulants.
Second, our study merely reports associations, instead of causality, between initiation of anticoagulants and patient characteristics. Third, as with other studies using claims data, we do not have information about laboratory results such as creatinine clearance or INR, so we cannot control for their effects on the initiation of drugs or calculate predictive scores for the risk of bleeding such as a HAS-BLED score. In addition, claims data do not contain information on the use of over-thecounter drugs. As a result, the proportion of patients using NSAIDs or antiplatelet agents may be underestimated.

Fourth, our study does not assess the effect of formulary placement, which may affect the initiation of dabigatran 
FIGURE 4 Comparing Odds of Initiating Dabigatran and Rivaroxaban

October 1, 2010-March 3, 2011

March 4, 2011-July 3, 2011

July 4, 2011-November 3, 2011

November 4, 2011-March 3, 2012

March 4, 2012-July 3, 2012

Midwest

South

West

Age 65-74

Age $>74$

Female

Black

Hispanic

Asian

Other

Death

Dual-eligibles

Non-dual LIS

Non-LIS in the coverage gap phase

Non-LIS in the catastrophic phase National PACE or employer-sponsored

AMI

Stroke

Kidney disease

$\mathrm{CHF}$

Hypertension

NSAID

Antiplatelet

\section{Dabigatran}

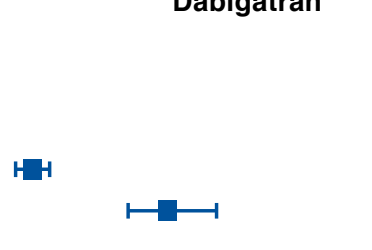

Rivaroxaban
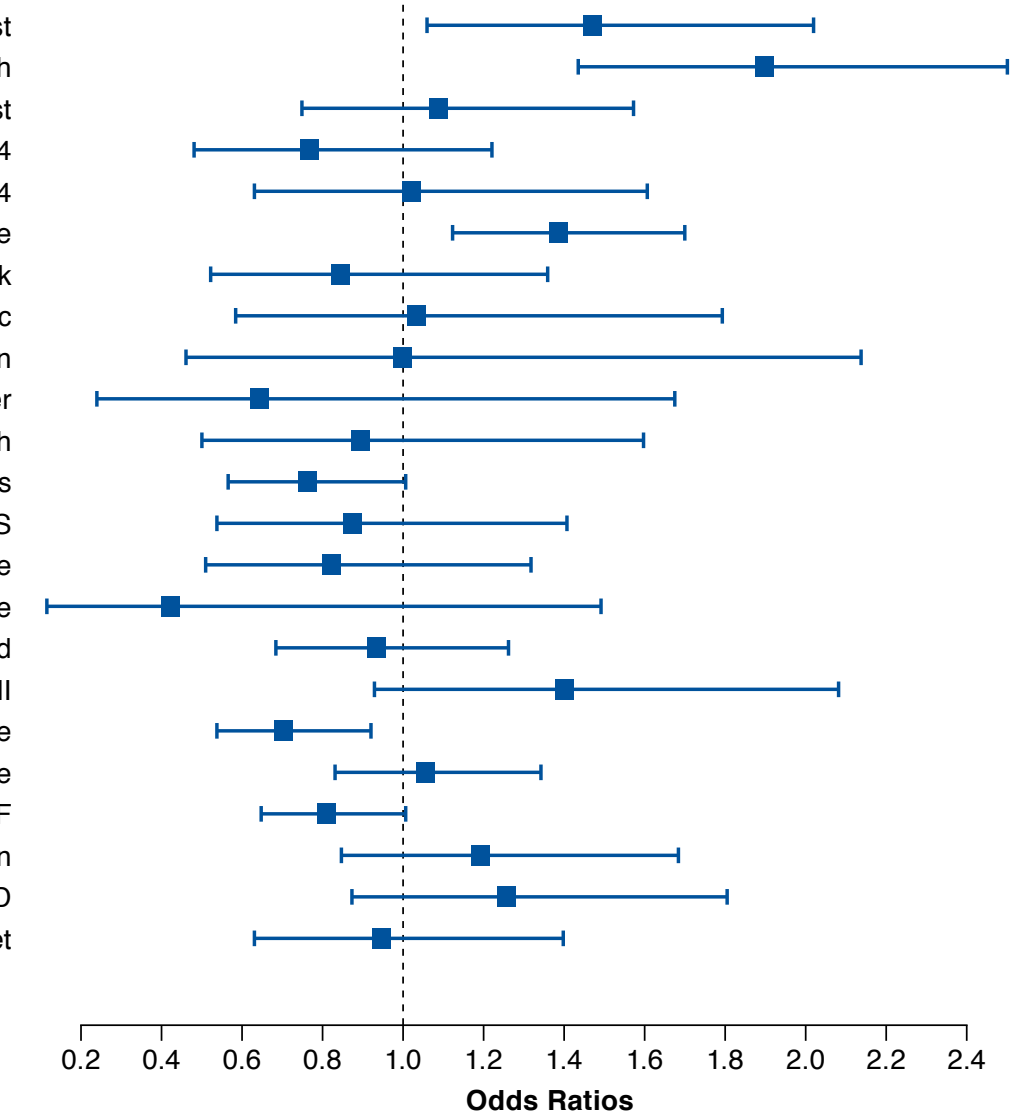

AMI = acute myocardial infarction; $C H F=$ congestive heart failure; LIS = low-income subsidy; NSAID = nonsteroidal anti-inflammatory drug.

compared with rivaroxaban. For example, if 1 plan placed dabigatran on the preferred brand tier and rivaroxaban in the nonpreferred brand tier, patients enrolled in that plan may be more likely to initiate dabigatran than rivaroxaban because they would have lower out-of-pocket expenses.

Finally, our study does not attempt to identify the effect of types of health care providers such as cardiologists and family medicine and internal medicine practitioners. Since health care providers prescribed oral anticoagulants according to their primary specialty, types of health care providers could potentially affect the initiation of NOACs.

\section{Conclusions}

This study evaluated how patient characteristics affect the initiation of anticoagulants among patients newly diagnosed with atrial fibrillation. We found that race, sex, type of Part D plans, and some clinical conditions are associated with the initiation of NOACs relative to warfarin. But patient demographic and clinical characteristics did not appear to affect which particular NOAC patients initiated. 


\section{Authors}

SEO HYON BAIK, PhD, is Staff Scientist, Lister Hill National Center for Biomedical Communications, National Library of Medicine, Bethesda, Maryland. INMACULADA HERNANDEZ, PharmD, is a PhD Student, and YUTING ZHANG, PhD, is Associate Professor and Director, Department of Health Policy and Management, University of Pittsburgh Graduate School of Public Health, Pittsburgh, Pennsylvania.

AUTHOR CORRESPONDENCE: Yuting Zhang, PhD, Department of Health Policy and Management, University of Pittsburgh, 130 De Soto St., Crabtree Hall A664, Pittsburgh, PA 15261. Tel.: 412.383.5340; Fax: 412.624.3146; E-mail: ytzhang@pitt.edu.

\section{DISCLOSURES}

This study received funding from the Commonwealth Foundation and Agency for Healthcare Research and Quality (No. R01 HS018657) and from the National Institute of Mental Health (No. R21 MH100721). The authors declare no conflict of interest. Biak was senior statistician in the Department of Health Policy and Management, University of Pittsburgh, at the time this study was conducted.

Baik, Hernandez, and Zhang contributed to the study concept and design. Baik took the lead in data collection, with assistance from Zhang and Hernandez. Data interpretation was performed by Baik, Hernandez, and Zhang. The manuscript was written and revised primarily by Baik, along with Hernandez and Zhang.

\section{REFERENCES}

1. January CT, Wann LS, Alpert JS, et al. 2014 AHA/ACC/HRS guideline for the management of patients with atrial fibrillation: a report of the American College of Cardiology/American Heart Association Task Force on Practice Guidelines and the Heart Rhythm Society. J Am Coll Cardiol. 2014;64(21): el-76. Available at: http://www.heartrhythmjournal.com/pb/assets/raw/ Health\%20Advance/journals/hrthm/AF_FT.pdf. Accessed December 5, 2015.

2. U.S. Food and Drug Administration. Drug approval packagePRADAXA (dabigatran etexilate mesylate) capsules. Approval date October 19, 2010. Available at: http://www.accessdata.fda.gov/drugsatfda_docs/ nda/2010/022512Origls000TOC.cfm. Accessed December 5, 2015.

3. U.S. Food and Drug Administration. Drug approval package-Xarelto (Rivaroxaban). Approval date November 4, 2011. Available at: http://www. accessdata.fda.gov/drugsatfda_docs/nda/2011/202439toc.cfm. Accessed December 5, 2015.

4. Connolly SJ, Ezekowitz MD, Yusuf S, et al. Dabigatran versus warfarin in patients with atrial fibrillation. N Eng J Med. 2009;361(12):1139-51. Available at: http://www.nejm.org/doi/full/10.1056/NEJMoa0905561. Accessed December 5, 2015.

5. Patel MR, Mahaffey KW, Garg J, et al. Rivaroxaban versus warfarin in nonvalvular atrial fibrillation. N Eng J Med. 2011;365(10):883-91. Available at: http://www.nejm.org/doi/full/10.1056/nejmoal009638. Accessed December 5, 2015.
6. U.S. Food and Drug Administration. Pradaxa (dabigatran): drug safety communication-lower risk for stroke and death, but higher risk for GI bleeding compared to warfarin. May 13, 2014. Available at: http://www.fda gov/safety/medwatch/safetyinformation/safetyalertsforhumanmedicalproducts/ucm397179.htm. Accessed December 5, 2015.

7. Hirsh J, Fuster V, Ansell J, Halperin JL; American Heart Association/ American College of Cardiology Foundation. American Heart Association/ American College of Cardiology Foundation guide to warfarin therapy. J Am Coll Cardiol. 2003;41(9):1633-52. Available at: http://content.onlinejacc.org/ article. aspx?articleid=1132287. Accessed December 6, 2015.

8. Freeman JV, Zhu RP, Owens DK, et al. Cost-effectiveness of dabigatran compared with warfarin for stroke prevention in atrial fibrillation. Ann Intern Med. 2011;154(1):1-11. Available at: http://annals.org/article. aspx?articleid=746681. Accessed December 5, 2015.

9. Lee S, Anglade MW, Pham D, Pisacane R, Kluger J, Coleman CI. Costeffectiveness of rivaroxaban compared to warfarin for stroke prevention in atrial fibrillation. Am J Cardiol. 2012;110(6):845-51. Available at: http:// www.sciencedirect.com/science/article/pii/S0002914912013410. Accessed December 5, 2015.

10. Hoadley J, Hargrave E, Merrell K, Summer L. Beneficiary-centered assignment for Medicare Part D: testing alternative approaches for ten beneficiary portfolios in five regions. Final report for the Medicare Payment Advisory Commission. January 15, 2009. Available at: http://ihcrp.georgetown.edu/pdfs/Beneficiary\%20Centered\%20Assignment $\% 20$ final $\% 20$ report\%201-15-09.pdf. Accessed December 6, 2015.

11. Chronic Condition Warehouse. Chronic condition reference list. Updated October 10, 2011. Available at: https://www.ccwdata.org/cs/groups/ public/documents/document/ccw_conditioncategories2011.pdf. Accessed December 6, 2015.

12. Centers for Medicare \& Medicaid Services. 2006-2011 model software/ ICD-9-CM mappings. Available at: https://www.cms.gov/Medicare/HealthPlans/MedicareAdvtgSpecRateStats/Risk-Adjustors-Items/Risk2006-2011. html?DLPage $=1 \&$ DLEntries $=25 \& D L S o r t=0 \& D L S o r t D i r=$ descending . Accessed December 13, 2015.

13. Centers for Medicare $\&$ Medicaid Services. Quick facts about Programs of All-Inclusive Care for the Elderly (PACE). Available at: https://www.medicare.gov/Pubs/pdf/11341.pdf. Accessed December 13, 2015.

14. AbuDagga A, Stephenson JJ, Fu AC, Kwong WJ, Tan H, Weintraub WS Characteristics affecting oral anticoagulant therapy choice among patients with non-valvular atrial fibrillation: a retrospective claims analysis. BMC Health Serv Res. 2014;14:310.

15. Desai NR, Krumme AA, Schneeweiss S, et al. Patterns of initiation of oral anticoagulants in patients with atrial fibrillation-quality and cost implications. Am J Med. 2014;127(11):1075-82. Available at: http://www.sciencedirect.com/science/article/pii/S0002934314003994. Accessed December 6, 2015.

16. Schoof N, Schnee J, Schneider G, et al. Characteristics of patients with non-valvular atrial fibrillation using dabigatran or warfarin in the U.S. Curr Med Res Opin. 2014;30(5):795-804.

17. Steinberg BA, Holmes DN, Piccini JP, et al. Early adoption of dabigatran and its dosing in US patients with atrial fibrillation: results from the outcomes registry for better informed treatment of atrial fibrillation. J Am Heart Assoc. 2013;2(6):e000535. 


\section{APPENDIX Definition of Chronic Conditions}

\begin{tabular}{|c|c|c|}
\hline Chronic Conditions & Valid ICD-9-CM Codes & $\begin{array}{l}\text { Number/Type of Claims to } \\
\text { Qualify }\end{array}$ \\
\hline Acute myocardial infarction & $\begin{array}{l}\text { DX } 410.01,410.11,410.21,410.31,410.41,410.51,410.61,410.71,410.81,410.91 \text { (ONLY } \\
\text { first or second DX on the claim) }\end{array}$ & $\begin{array}{l}\text { At least } 1 \text { inpatient claim } \\
\text { with DX codes prior to atrial } \\
\text { fibrillation diagnosis }\end{array}$ \\
\hline Chronic kidney disease & $\begin{array}{l}\text { DX 016.00, } 016.01,016.02,016.03,016.04,016.05,016.06,095.4,189.0,189.9,223.0, \\
236.91,249.40,249.41,250.40,250.41,250.42,250.43,271.4,274.10,283.11,403.01, \\
403.11,403.91,404.02,404.03,404.12,404.13,404.92,404.93,440.1,442.1,572.4, \\
580.0,580.4,580.81,580.89,580.9,581.0,581.1,581.2,581.3,581.81,581.89,581.9, \\
582.0,582.1,582.2,582.4,582.81,582.89,582.9,583.0,583.1,583.2,583.4,583.6, \\
583.7,583.81,583.89,583.9,584.5,584.6,584.7,584.8,584.9,585.1,585.2,585.3, \\
585.4,585.5,585.6,585.9,586,587,588.0,588.1,588.81,588.89,588.9,591,753.12, \\
753.13,753.14,753.15,753.16,753.17,753.19,753.20,753.21,753.22,753.23,753.29, \\
794.4 \text { (any DX on the claim) }\end{array}$ & $\begin{array}{l}\text { At least } 1 \text { inpatient, SNF, or } \\
\text { HHA claim or } 2 \text { HOP or carrier } \\
\text { claims with DX codes prior to } \\
\text { atrial fibrillation diagnosis }\end{array}$ \\
\hline Diabetes & 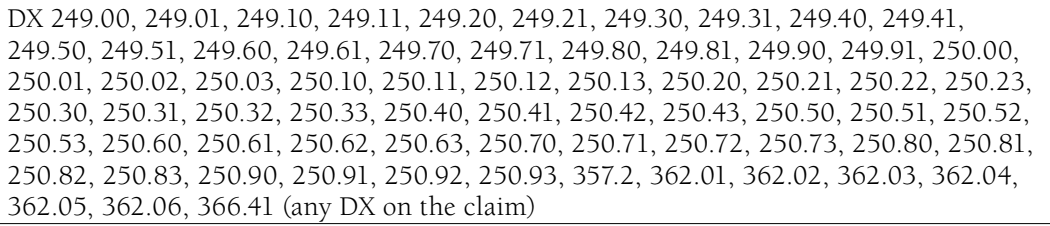 & $\begin{array}{l}\text { At least } 1 \text { inpatient, SNF, or } \\
\text { HHA claim or } 2 \text { HOP or carrier } \\
\text { claims with DX codes prior to } \\
\text { atrial fibrillation diagnosis }\end{array}$ \\
\hline Heart failure & $\begin{array}{l}\text { DX } 398.91,402.01,402.11,402.91,404.01,404.03,404.11,404.13,404.91,404.93 \\
428.0,428.1,428.20,428.21,428.22,428.23,428.30,428.31,428.32,428.33,428.40 \\
428.41,428.42,428.43,428.9 \text { (any DX on the claim) }\end{array}$ & $\begin{array}{l}\text { At least } 1 \text { inpatient, HOP, or } \\
\text { carrier claim with DX codes } \\
\text { prior to atrial fibrillation } \\
\text { diagnosis }\end{array}$ \\
\hline Hypertension & $\begin{array}{l}\text { DX 362.11, 401.0, 401.1, 401.9, 402.00, 402.01, 402.10, 402.11, 402.90, 402.91, 403.00, } \\
403.01,403.10,403.11,403.90,403.91,404.00,404.01,404.02,404.03,404.10,404.11, \\
404.12,404.13,404.90,404.91,404.92,404.93,405.01,405.09,405.11,405.19,405.91, \\
405.99,437.2 \text { (any DX on the claim) }\end{array}$ & $\begin{array}{l}\text { At least } 1 \text { inpatient, SNF, HHA, } \\
\text { or } 2 \text { HOP or carrier claims } \\
\text { with DX codes prior to atrial } \\
\text { fibrillation diagnosis }\end{array}$ \\
\hline Hypothyroidism & DX 244.0, 244.1, 244.2, 244.3, 244.8, 244.9 (any DX on the claim) & $\begin{array}{l}\text { At least } 1 \text { inpatient, SNF, HHA, } \\
\text { or } 2 \text { HOP or carrier claims } \\
\text { with DX codes prior to atrial } \\
\text { fibrillation diagnosis }\end{array}$ \\
\hline Ischemic heart disease & $\begin{array}{l}\text { DX 410.00, 410.01, 410.02, 410.10, 410.11, 410.12, 410.20, 410.21, 410.22, 410.30, 410.31, } \\
410.32,410.40,410.41,410.42,410.50,410.51,410.52,410.60,410.61,410.62,410.70, \\
410.71,410.72,410.80,410.81,410.82,410.90,410.91,410.92,411.0,411.1,411.81, \\
411.89,412,413.0,413.1,413.9,414.00,414.01,414.02,414.03,414.04,414.05,414.06, \\
414.07,414.12,414.2,414.3,414.8,414.9 \text { (any DX on the claim) }\end{array}$ & $\begin{array}{l}\text { At least } 1 \text { inpatient, SNF, HHA, } \\
\text { HOP, or carrier claim with DX } \\
\text { codes prior to atrial fibrillation } \\
\text { diagnosis }\end{array}$ \\
\hline $\begin{array}{l}\text { Stroke/transient ischemic } \\
\text { attack }\end{array}$ & $\begin{array}{l}\text { DX 430, 431, 433.01, 433.11, 433.21, 433.31, 433.81, 433.91, 434.00, 434.01, 434.10, } \\
434.11,434.90,434.91,435.0,435.1,435.3,435.8,435.9,436,997.02 \text { (any DX on the } \\
\text { claim) }\end{array}$ & $\begin{array}{l}\text { At least } 1 \text { inpatient claim or } 2 \\
\text { HOP or carrier claims with DX } \\
\text { codes prior to atrial fibrillation } \\
\text { diagnosis }\end{array}$ \\
\hline
\end{tabular}

DX= diagnosis; HHA = home health agency; HOP=hospital outpatient; ICD-9-CM=International Classification of Diseases, Ninth Revision, Clinical Modification; SNF = skilled nursing facility. 